\title{
Overview of the 63rd session of the WHO Regional Committee for the Eastern Mediterranean
}

\author{
Ala Alwan ${ }^{1}$
}

The 63rd session of the Regional Committee for the Eastern Mediterranean was held at the WHO Regional Office in Cairo, Egypt, 3-6 October 2016, attended by representatives of the 22 Members of the Committee, along with observers from other UN and intergovernmental organizations and nongovernmental organizations. The achievements, challenges and way forward for $\mathrm{WHO}$ in the Region formed much of the agenda, given that the overarching goal of WHO's work with Member States since 2012 focused upon the five strategic priorities, namely: health systems strengthening towards universal health coverage; maternal and child health; noncommunicable diseases; health security and communicable diseases; and emergency preparedness and response. The Regional Director's Annual Report, which was presented and discussed during the Regional Committee, emphasized the main achievements with respect to these strategic priorities for WHO's work in the Region.

An update on the implementation of the International Health Regulations (IHR 2005) was brought to the attention of the Regional Committee through the work of the independent regional assessment commission, established in accordance with resolution EM/RC62/R.3 to assess and advise Member States. It made a number of recommendations to countries to accelerate IHR implementation in the Region, following joint external evaluation assessments conducted in six countries in the Region, with nine more planned up to April 2017. The Regional Committee was also invited to provide comments on the $\mathrm{WHO}$ draft global implementation plan for the recommendations of the Review Committee on the Role of the IHR in the Ebola Outbreak and Response. As part of its conclusion, the Regional Committee highlighted the importance of compliance with the regulations and maintaining core capacities, along with the key role of multisectoral coordination in this, and the need to focus on mass gatherings.

Improving access to assistive technology is an area that needs addressing in light of the protracted crises in the Region and resulting numbers of people with disabilities. The Regional Committee discussed the need to increase access to assistive technology and ensure its integration in universal health coverage, and suggested the need to redefine assistive technology on a broader basis and to change the way assistive products are designed, distributed and financed. This point was emphasized by several representatives present who drew attention to multisectoral collaboration as critical for the identification of needs and the provision of assistive products, as well as the role of primary health care in needs identification, provision and follow-up of utilization. Moreover, attention was drawn to the importance of a legal framework for the adoption of policies and programmes related to assistive technology, and for capacitybuilding and mechanisms to provide assistive products at affordable cost. To this end, the Regional Committee urged the necessity for cooperation between Member States to develop an evidence-based integrated policy to improve access to assistive technology for all as an essential component of health service delivery systems, supported by adequate financing, and to conduct a needs assessment using appropriate WHO tools to inform adequate planning of services.

Public health deliverance and promotion of universal health coverage clearly requires the support of effective and adequate blood transfusion and health laboratory systems. The Regional Committee discussed the proposed strategic frameworks which aimed at enabling countries to ensure that their national laboratory systems were wellcoordinated, sustainable, accessible for all and able to generate safe, reliable and timely results for public health purposes and patient care. In addition, discussions covered the development and strengthening of their national blood systems to ensure the continuity, sufficiency, sustainability and security of national supplies of safe and efficacious blood and blood components to meet national needs. The need to integrate laboratory and blood transfusion services within vertical health programmes, and support in quality assurance and laboratory accreditation were highlighted, and not excluding the importance of strengthening the public health and epidemic preparedness roles of health laboratory services. The proposed strategic frameworks were endorsed by the Regional Committee, which asked Member States to develop national 
plans accordingly and requested $\mathrm{WHO}$ to provide support in this regard.

Advancing family practice towards universal health coverage in the Region received notable attention by the Regional Committee, which discussed the need for comprehensive and sustainable national policies and programmes on family practice, together with a framework for action for Member States and WHO. However, representatives highlighted some of the constraints to scaling up family practice in the Region and stressed the need for reform of medical education curricula, an operational guide linking family practice with secondary care, and standardization of bridging programmes as a transitional arrangement to upgrading general physicians to family physicians.
Thus, Member States were encouraged to incorporate the family practice approach into their primary health care services as an overarching strategy to advance towards universal health coverage. In addition, strengthening the capacity of family medicine departments in public health facilities and medical education institutions was highlighted in order to increase the number of family physicians and to establish bridging programmes for general physicians.

WHO's work in the Region has had an important and effective impact, supporting the needs and rights of citizens to universal health coverage in what continues to be very difficult times due to ongoing regional conflicts exacerbating health services degradation. Looking forward, the Regional Committee supported WHO in providing further technical guidance in the Region for assessment of emergency care services; organizing an intercountry consultative meeting on genetic and congenital disorders; and submitting an evidencebased plan of action for the regional implementation of the global roadmap to address the health impacts of indoor and outdoor air pollution to the $64^{\text {th }}$ session of the Regional Committee.

We thank representatives from the 22 Members of the Regional Committee and observers for their contributions to the work WHO undertakes in the Region with dedication, and the Committee's recognition of the valuable developments and initiatives that WHO has brought to fruition in the Eastern Mediterranean Region. 